\title{
Mandenkan
}

MANDENIKAN Bulletin semestriel d'études linguistiques mandé

49 | 2013

Le maninka du Niokolo

\section{Formes verbales dépendantes}

Section 7

Denis Creissels

\section{(2) OpenEdition}

Journals

Édition électronique

URL : https://journals.openedition.org/mandenkan/603

DOI : $10.4000 /$ mandenkan.603

ISSN : 2104-371X

Éditeur

Llacan UMR 8135 CNRS/Inalco

Édition imprimée

Date de publication : 1 juin 2013

Pagination : 60-62

ISSN : 0752-5443

Référence électronique

Denis Creissels, «Formes verbales dépendantes », Mandenkan [En ligne], 49 | 2013, mis en ligne le 25 avril 2014, consulté le 16 janvier 2023. URL : http://journals.openedition.org/mandenkan/603 ; DOI : https://doi.org/10.4000/mandenkan.603

Ce document a été généré automatiquement le 16 janvier 2023.

\section{(c) $)(1)(2)$}

Creative Commons - Attribution - Pas d'Utilisation Commerciale - Partage dans les Mêmes Conditions 4.0 International - CC BY-NC-SA 4.0

https://creativecommons.org/licenses/by-nc-sa/4.0/ 


\section{Formes verbales dépendantes}

\section{Section 7}

\section{Denis Creissels}

\subsection{L'infinitif en 'ka 'ke}

1 L'infinitif en ' $k a \sim$ ' $k e$ s'emploie pour topicaliser le groupe verbal, comme à l'ex. (51), et dans la complémentation de verbes aspectuels ou modaux, comme à l'ex. (52). Les deux variantes ' $k a$ et ' $k e$ semblent totalement équivalentes.

\begin{tabular}{|l|l|l|l|l|l|l|l|}
\hline (51) & a. & Ká & fula-káy-o & káray, & a & kóle-yáa-ta & lée. \\
\hline & & INF & peul-langue-D & apprendre & 3SG & être_difficile-ABSTR-ACPP & FOC \\
\hline & & \multicolumn{4}{|l}{ 'Apprendre le peul, c'est difficile.' } \\
\hline
\end{tabular}

\begin{tabular}{|l|l|l|l|l|l|l|l|l|}
\hline & b. & Ká & kuma & dii-máa & fo, & wóo & mà & kóle. \\
\hline & & INF & parole & être_agréable-SELECT.D & dire & DEM & ACPN & être_difficile \\
\hline & \multicolumn{6}{|l}{} \\
\hline
\end{tabular}

\begin{tabular}{|l|l|l|l|l|l|l|l|l|l|l|}
\hline (52) & a. & Fondinkée & lu & nin & $\hat{i}$ & sii-ta & ján & doo & ma, \\
\hline & & adolescent.D & PL & si & 3 PL & arriver-D & stade & INDEF & OBL \\
\hline & & 'Les adolescents, lorsqu'ils atteignent un certain stade, & \\
\hline & & $i$ & lafii-ta & & $k e$ & búla & kéebáa & lu & kay. \\
\hline & & 3PL & vouloir-ACPP & INF & s'installer & adulte.D & PL & sur & \\
\hline
\end{tabular}


ils veulent intégrer le groupe des adultes.'

\begin{tabular}{|l|l|l|l|l|l|l|l|l|}
\hline & b. & I & măy & $k a \eta$ & $k a ́$ & $i$ & teerikée & jámfaa. \\
\hline & & 3PL & ACPN & devoir & INF & 2SG & ami.D & trahir \\
\hline & \multicolumn{6}{|c|}{ 'Tu ne dois pas trahir ton ami.' } \\
\hline
\end{tabular}

\begin{tabular}{|c|c|c|c|c|c|c|c|c|}
\hline c. & $A$ & be & $a$ & fee & $k a ́$ & taya & ñá $\gamma a$ & to. \\
\hline & $3 S G$ & COPLOC & $3 S G$ & OBL & INF & aller & circoncision.D & LOC \\
\hline & 110 & $c$ & & & & & & \\
\hline
\end{tabular}

\subsection{L'infinitif en - 'la}

2 Comme en mandinka, dans la complémentation des verbes aspectuels et modaux, l'infinitif en ' $k a \sim$ ' $k e$ est en concurrence avec une forme obtenue par suffixation de - 'la au verbe.

\begin{tabular}{|l|l|l|l|l|l|l|}
\hline (53) & a. & A & may & són & boor-óo & táa-la. \\
\hline & & 3SG & ACPN & accepter & médicament-D & prendre-INF \\
\hline & & \multicolumn{4}{|c|}{ 'Il ne veut pas prendre le médicament.' } \\
\hline
\end{tabular}

\begin{tabular}{|l|l|l|l|l|l|l|l|}
\hline & b. & A & man & són & & $\eta$ & déemaía-la. \\
\hline & & $3 S G$ & ACPN & accepter & $1 S G$ & aider-INF & \\
\hline & & \multicolumn{6}{|l|}{ 'Il n'est pas d'accord pour m'aider.' } \\
\hline
\end{tabular}

\begin{tabular}{|l|l|l|l|l|l|l|l|l|}
\hline & c. & $\eta$ & be & láfii-rin & wóo & lée & kácaa-la & bíi. \\
\hline & & $1 S G$ & COPLOC & vouloir-RES & DEM & FOC & discuter-INF & aujourd'hui \\
\hline & \multicolumn{6}{|l|}{ 'C'est de cela que je veux discuter aujourd'hui.' } \\
\hline
\end{tabular}

3 L'infinitif en - 'la a aussi été observé dans une construction où il exprime une prédication portant sur l'objet du verbe je 'voir'.

\begin{tabular}{|l|l|l|l|l|l|l|l|l|l|}
\hline (54) & Nì & $i$ & ye & mús-ǒo & mèn & je & sóyon-na & gánkurán-o & to, \\
\hline
\end{tabular}




\begin{tabular}{|l|l|l|l|l|l|l|l|l|l|}
\hline & si & 2SG & ACPP & femme-D & REL & voir & initier-D & gankourang-D & LOC \\
\hline \multicolumn{7}{|l|}{ 'Si tu vois une femme initiée au gankourang, } \\
\hline & $i$ & sé & $a$ & tára & $a$ & támbi-'-ta & lée & kée-la-láa & ma. \\
\hline & 2SG & POTP & $35 G$ & trouver & $35 G$ & passer-ACPP & FOC & homme-avec-coucher.D & OBL \\
\hline
\end{tabular}

\subsection{L'infinitif nu}

Une forme d'infinitif qui coïncide avec la base verbale nue s'emploie comme complément de taya 'aller' et náa 'venir'.

\begin{tabular}{|l|l|l|l|l|l|l|l|l|}
\hline (55) & a. & Dindiy-o & lu & tara-ta & ñins-ǒo & lée & lu & biti. \\
\hline & & enfant-D & PL & aller-ACPP & vache-D & FOC & PL & traire \\
\hline & \multicolumn{6}{|l}{} \\
\hline
\end{tabular}

\begin{tabular}{|c|c|c|c|c|c|c|c|}
\hline b. & $A$ & náa-ta & ñin & kumá- $\eta$ & $f_{0}$ & $y$ & yey. \\
\hline & $3 S G$ & venir-ACPP & DEM & parole-DEF & dire & $1 S G$ & BEN \\
\hline & \multicolumn{7}{|c|}{ 'Il est venu me parler de ça.' } \\
\hline
\end{tabular}

\subsection{Le participe résultatif}

5 Le suffixe - 'riy - 'lig - 'diy qui caractérise cette forme a déjà été mentionnée parmi les suffixes dérivatifs, car la forme qu'il permet d'obtenir a des emplois où on peut simplement la décrire comme adjectif dérivé de verbe. Mais cette forme a aussi d'autres emplois, dans lesquels elle est la tête d'un groupe verbal, dans la prédication analytique (cf. 9.1) ainsi que dans la prédication seconde (cf. 17).

\subsection{Le gérondif}

6 L'adjonction du suffixe -tóo à une base verbale donne une forme désignée ici comme gérondif, qui s'emploie dans la prédication seconde (cf.17) ainsi que dans une construction prédicative analytique (cf. 9.4) 


\subsection{La forme progressive}

7 Caractérisée par un suffixe $-k a-i ́ n$, cette forme s'utilise exclusivement comme complément de la copule locative, dans une construction exprimant l'aspect progressif (cf. 9.2).

\section{AUTEUR}

\section{DENIS CREISSELS}

Université de Lyon

Denis.Creissels@univ-lyon2.fr 